\title{
miR-802 inhibits the epithelial-mesenchymal transition, migration and invasion of cervical cancer by regulating BTF3
}

\author{
XIUHUI WU ${ }^{1 *}$, LENG LIU $^{1 *}$ and HONGXIA ZHANG ${ }^{2}$ \\ ${ }^{1}$ Department of Gynecology, Jingmen No.1 People's Hospital, Jingmen, Hubei 448000; \\ ${ }^{2}$ Department of Breast Surgery, Xiantao First People's Hospital, Xiantao, Hubei 433000, P.R. China
}

Received July 3, 2019; Accepted March 27, 2020

DOI: $10.3892 / \mathrm{mmr} .2020 .11267$

\begin{abstract}
MicroRNA (miR)-802 has been discovered to be involved in the occurrence and development of numerous types of tumor; however, studies into the role of miR-802 in cervical cancer are limited. Therefore, the present study aimed to investigate the regulatory effects of miR-802 in cervical cancer cells. miR-802 expression levels in cervical cancer tissue and cells were analyzed using reverse transcription-quantitative (RT-q) PCR, a dual-reporter luciferase activity assay was used to identify the direct target gene of miR-802, and RT-qPCR and western blotting were performed to determine the relationship between miR-802 and basic transcription factor 3 (BTF3). Cell viability, and migration and invasion were analyzed using Cell Counting Kit- 8 and Transwell assays, respectively. Finally, the expression levels of metastasis-associated proteins, $\mathrm{N}$-cadherin and E-cadherin, were determined using RT-qPCR and western blotting. Decreased expression levels of miR-802 were found in cervical cancer tissues and cells, and the overexpression of miR-802 inhibited cell viability, migration and invasion. Moreover, miR-802 was discovered to directly target BTF3 to inhibit its expression. Notably, the overexpression miR-802 markedly reversed the promotive effect of BTF3 on cell viability, in addition to the migratory and invasive abilities of the cells. Simultaneously, the overexpression of miR-802 significantly suppressed epithelial-mesenchymal transition, and the expression levels of matrix metallopeptidase (MMP)2 and MMP9 in cells through regulating BTF3. In conclusion, the present study revealed that miR-802 may suppress cervical cancer progression by decreasing BTF3 expression levels, indicating that it may represent a potential therapeutic target for the treatment and prognosis of patients with cervical cancer.
\end{abstract}

Correspondence to: Dr Hongxia Zhang, Department of Breast Surgery, Xiantao First People's Hospital, 29 Middle Section of Mianzhou Avenue, Xiantao, Hubei 433000, P.R. China

E-mail: zhanghonhxia_hx@163.com

*Contributed equally

Key words: microRNA-802, cervical cancer, basic transcription factor 3, epithelial-mesenchymal transition, viability

\section{Introduction}

Cervical cancer is a common malignancy of the female reproductive system, demonstrating the second highest morbidity rate among female malignancies worldwide $(1,2)$. Chemotherapy, radiotherapy and surgical treatment are the standard methods for treating cervical cancer; however, its 5-year survival rate remains low, with lymph node and distant metastasis being the contributing factors leading to treatment failure $(3,4)$. Therefore, to provide potential targets for subsequent individualized treatment, it is important to study the molecular mechanisms of the occurrence and development of cervical cancer.

MicroRNAs (miRNA/miR) are a class of non-coding RNAs that exist in numerous biological genomes, which have the function of sequence-specific regulation over gene expression $(5,6)$. The regulatory role of miRNAs mainly occurs at the post-transcriptional level, as miRNAs inhibit protein translation and/or promote the degradation of $\operatorname{mRNA}(7,8)$. miRNAs serve important roles not only in embryonic formation and the growth and development of organisms, but also in cell proliferation, differentiation, apoptosis and the occurrence of several human diseases $(9,10)$. Previous studies have demonstrated that abnormal miRNA expression critically affected tumor growth, invasion and apoptosis $(11,12)$. In addition, miR-802 was also revealed to be involved in tumorigenesis and development, whereby it served distinct roles in different types of tumor $(13,14)$. However, studies into the role of miR-802 in cervical cancer are limited. In one study, Zhang et al (15) found that miR-802 inhibited cell proliferation and induced apoptosis in human cervical cancer by targeting serine/arginine-rich splicing factor 9 . Thus, the present study further investigated the effect of miR-802 on the biological characteristics of cervical cancer cells.

Studies have reported that one miRNA can simultaneously regulate hundreds of target genes, including transcription factors, cytokines and receptors (16-18). The regulatory networks between miRNAs and their target genes have been revealed to be involved in the occurrence and development of tumors (19); miRNAs affect cell proliferation, apoptosis, invasion, angiogenesis, metastasis and other biological behaviors by regulating the mRNA translational levels of target genes, which subsequently leads to the occurrence and development of tumors (20). In the current study, TargetScan 
7.2 software was used to predict the target genes of miR-802; the results revealed that miR-802 has a complementary binding site in the 3'-untranslated region (UTR) of the basic transcription factor 3 (BTF3) gene. Therefore, the relationship between miR-802 and BTF3 was further investigated to determine the role of miR-802 in the occurrence and development of cervical cancer.

\section{Materials and methods}

Patient studies. Cervical cancer tissue and adjacent tissue samples were obtained from 40 female patients (age, $43.57 \pm 2.79$ ) with cervical cancer who attended Jingmen First People's Hospital between January 2018 and May 2019. The tissue samples were maintained in liquid nitrogen and stored at $-80^{\circ} \mathrm{C}$. Exclusion criteria: i) other gynaecological diseases; ii) severe internal and external diseases; iii) history of chemoradiotherapy; and iv) history of pelvic organ surgery. All research subjects provided written informed consent and the study was approved by the Ethics Committees of Jingmen No. 1 People's Hospital.

Cell culture. The human endometrial epithelial cell (Ect1/E6E7) line were purchased from American Type Culture Collection. The cervical cancer cell lines (HeLa, C-33 A, SiHa and ME-180) were purchased from the American Type Culture Collection. All the cells were cultured in DMEM (cat. no. D0819; Sigma-Aldrich; Merck KGaA), supplemented with $10 \%$ FBS (Gibco; Thermo Fisher Scientific, Inc.) at $37^{\circ} \mathrm{C}$ in a $5 \% \mathrm{CO}_{2}$ atmosphere.

Cell transfection. Among the cervical cancer cell lines, as miR-802 was expressed at the lowest levels in SiHa cells, $\mathrm{SiHa}$ cells were used in the following experiments. For transfection, $2 \mathrm{ml}$ cell solution, containing $1 \times 10^{6}$ cells/well and culture medium, was plated into 6 -well plates and incubated at $37^{\circ} \mathrm{C}$ in a $5 \% \mathrm{CO}_{2}$ atmosphere until the cell confluence reached 40-60\%. To prepare A transdye, 20 pmol miR-802 mimic (forward, 5'-UCAGUAACAAAGAUUCAUCCUUGU-3' and reverse, 5'-ACAAGGAUGAAUCUUUGUUACUGA-3'; Shanghai GenePharma Co., Ltd.), the negative control for the mimic (NC-mimic; a non-targeting sequence; forward, 5'-UUUUACUACACAAAAGUACUG-3' and reverse, 5'-CAGUACUUUUGUGUAGUACAAA-3'; Shanghai GenePharma Co., Ltd.), BTF3 overexpression plasmid (pWZL-BTF3; Shanghai GenePharma Co., Ltd.) or the empty plasmid (pWZL-Blast; pWZL-NC; Shanghai GenePharma Co., Ltd.) were dissolved in $50 \mu 1$ DMEM (Hyclone; GE Healthcare Life Sciences) and fully mixed. To prepare B transdye, $1 \mu 1$ Lipofectamine ${ }^{\circledR} 2000$ reagent (Invitrogen; Thermo Fisher Scientific, Inc.) was dissolved in $50 \mu \mathrm{l}$ DMEM, incubated for 15 min at room temperature and then mixed with A transdye. This solution $(50 \mu \mathrm{l})$ was subsequently added into each well of the plate and incubated at $37^{\circ} \mathrm{C}$ with $5 \% \mathrm{CO}_{2}$. Following $24 \mathrm{~h}$ of transfection, the medium was changed and the cells were collected after culture for $72 \mathrm{~h}$.

Experimental grouping. To investigate the effects of miR-802 on the viability, migration and invasion of cervical cancer cells, the cells were divided into three groups: Control (untreated cells), NC-mimic (cells transfected with the NC-mimic) and miR-802 mimic (cells transfected with the miR-802 mimic) group. To determine the effects of BTF3 on the viability, migration and invasion of cervical cancer cells, the cells were divided into five groups: Control (untreated cells), pWZL-NC + NC-mimic (cells co-transfected with empty plasmid and NC-mimic), pWZL-BTF3 + NC-mimic (cells co-transfected with BTF3 overexpression plasmid and NC-mimic), pWZL-BTF3 + miR-802 mimic (cells co-transfected with BTF3 overexpression plasmid and miR-802 mimic) and miR-802 mimic (cells transfected with miR-802 mimic) group.

Dual-luciferase activity assay. The target-binding region of miR-802 and BTF3 was predicted using TargetScan (version 7.2; www.targetscan.org/vert_72/). For the dual-luciferase reporter assays, the 3 '-UTR of BTF3 containing miR-802 binding sites was inserted into a pmirGLO dual-luciferase vector (Promega Corporation) to generate wild-type (WT) BTF3-3'-UTR. The mutant (mut) 3' UTR of BTF3 was synthesized using a Site-Directed Mutagenesis kit (Thermo Fisher Scientific, Inc.) and inserted into the pmirGLO dual-luciferase vector to generate BTF3-3'-UTR-mut. The pmirGLO vector containing WT or mut BTF3 3'-UTR was co-transfected with the miR-802 mimic or NC-mimic into SiHa cells using Lipofectamine ${ }^{\circledR} 2000$ reagent (Invitrogen; Thermo Fisher Scientific, Inc.). Following incubation for 48 h, the relative luciferase activity in the cells was measured using a Dual-Luciferase Reporter Assay kit (Promega Corporation) according to the manufacturer's protocol. Firefly luciferase activities were normalized to Renilla luciferase activities.

Cell migration assay. Following $24 \mathrm{~h}$ of transfection, $\mathrm{SiHa}$ cells were resuspended in DMEM without FBS until the cell density reached $1 \times 10^{6}$ cells $/ \mathrm{ml}$. Then, $100 \mu \mathrm{l}$ cell suspension was plated into the upper chambers of the Transwell plate (Corning, Inc.) and cultured at $37^{\circ} \mathrm{C}$ for $6 \mathrm{~h}$. DMEM with $10 \%$ FBS was plated into the lower chamber. Following the incubation, the non-migratory cells were removed with a cotton swab and the migratory cells were washed three times with PBS, and fixed with 4\% formaldehyde (cat. no. P0099; Beyotime Institute of Biotechnology) for $20 \mathrm{~min}$ at room temperature and with 1\% Triton X-100 (cat. no. P0096; Beyotime Institute of Biotechnology) for $5 \mathrm{~min}$ at room temperature. After washing the cells three times with PBS, the cells were stained with hematoxylin (cat. no. C0107; Beyotime Institute of Biotechnology) for $20 \mathrm{~min}$ at room temperature and then washed under running water. Stained cells were counted in five randomly selected fields using a light microscope (magnification, $\mathrm{x} 200$; Olympus Corporation); the mean number of cells per field of view was calculated.

Cell invasion assay. Following $24 \mathrm{~h}$ of transfection, SiHa cells were resuspended in DMEM without FBS until the cell density reached $1 \times 10^{6}$ cells $/ \mathrm{ml}$. The upper chambers of the Transwell plates (pore size, $8.0 \mu \mathrm{m}$; Corning, Inc.) were precoated with $50 \mu \mathrm{l}$ Matrigel (BD Biosciences) for $2 \mathrm{~h}$ at $4^{\circ} \mathrm{C}$. Then, the cell suspension was subsequently plated into the upper chamber of the Transwell plate. DMEM, supplemented with 10\% FBS was plated in the lower chambers. Following incubation for $24 \mathrm{~h}$ at $37^{\circ} \mathrm{C}$, the invasive cells in the lower chamber were fixed in 
Table I. Primers used for the reverse transcription-quantitative PCR.

\begin{tabular}{ll}
\hline Gene & \multicolumn{1}{c}{ Primer sequence $\left(5^{\prime} \rightarrow 3^{\prime}\right)$} \\
\hline N-cadherin & F: ATGAAGAAGGTGGAGGAGA \\
E-cadherin & F: AGATCGGACCGGATACT \\
& R: TGGAGGAATTCTTGCTTTGC \\
Basic transcription factor 3 & F: AGCTTGGTGCGGATAGTCTGA \\
MMP2 & R: GTGCTTTTCCATCCACAGATTG \\
& F: TCTCCTGACATTGACCTTGGC \\
MMP9 & R: CAAGGTGCTGGCTGAGTAGATC \\
FAPDH & F: TTGACAGCGACAAGAAGTGG \\
R: GCCATTCACGTCGTCCTTAT & F: GCTGGCGCTGAGTACGTCGTGGAGT \\
microRNA-802 & R: CACAGTCTTCTGGGTGGCAGTGATGG \\
U6 & F: CGTTGTGTAGCTTATCAGACTG \\
& R: AATGGTTGTTCTCCACACTCTC \\
\hline
\end{tabular}

MMP, matrix metallopeptidase; F, forward; R, reverse.

$5 \%$ gluteraldehyde for $30 \mathrm{~min}$ at room temperature and stained with $0.5 \%$ crystal violet for $20 \mathrm{~min}$ at room temperature, while the non-invasive cells remaining in the upper chamber were removed using a cotton swab. Stained cells were observed in three randomly selected fields of view using a light microscope (magnification, $\mathrm{x} 200$ ).

Cell viability. SiHa cells $\left(2 \times 10^{4}\right.$ cells/well) were plated into 96-well plates in DMEM containing 10\% FBS and cultured for $24 \mathrm{~h}$ at room temperature. Subsequently, $10 \mu \mathrm{l}$ Cell Counting Kit-8 (cat. no. 96992; Sigma-Aldrich; Merck KGaA) solution was added into each well, according to the manufacturer's protocol, and incubated for $4 \mathrm{~h}$ at $37^{\circ} \mathrm{C}$. The absorbance at $450 \mathrm{~nm}$ was used a Multiskan GO microplate reader (Shanghai Bajiu Industrial Co., Ltd.). The experiment was conducted in triplicate and the average absorbance value was calculated.

Western blot analysis. SiHa cells were washed twice with cold PBS and total protein was extracted from the cells using RIPA lysis buffer (Cell Signaling Technology, Inc.). Total protein was quantified using the bicinchoninic acid protein assay kit (Pierce; Thermo Fisher Scientific, Inc.). The extracted protein was denatured for $5 \mathrm{~min}$ at $100^{\circ} \mathrm{C}$, and $50 \mu \mathrm{g} / \mu \mathrm{l}$ protein/lane was separated via $15 \%$ SDS-PAGE. The separated proteins were subsequently transferred onto PVDF membranes and blocked with $5 \%$ milk at room temperature for $1 \mathrm{~h}$. The membranes were incubated with the following primary antibodies at $4^{\circ} \mathrm{C}$ overnight: Anti-N-cadherin (1:1,000; cat. no. ab18203; Abcam), anti-BTF3 (1:1,000; cat. no. ab203517; Abcam), anti-matrix metallopeptidase (MMP) 9 (1:1,000; cat. no. ab73734; Abcam), anti-MMP2 (1:1,000; cat. no. ab37150; Abcam), anti-E-cadherin (1:1,000; cat. no. ab40772; Abcam) and anti-GAPDH (1:2,000; cat. no. ab8245; Abcam). Following the primary antibody incubation, the membranes were incubated with goat anti-mouse or goat anti-rabbit IgG $(\mathrm{H}+\mathrm{L})$ horseradish peroxidase-conjugated secondary antibodies (cat. nos. SA00001-1 and SA00001-2; 1:2000; ProteinTech Group, Inc.) for $2 \mathrm{~h}$ at room temperature and then washed with PBS three times. Protein bands were visualized using an ECL western blotting kit (cat. no. 93-K820-500; Hangzhou Multi Sciences (Lianke) Biotech Co., Ltd.) and the expression levels were semi-quantified using ImageJ v4.7 software (National Institutes of Health).

Reverse transcription-quantitative PCR (RT-qPCR). Total RNA was extracted from cells using TRIzol ${ }^{\circledR}$ reagent (Invitrogen; Thermo Fisher Scientific, Inc.) and the concentration of RNA was measured using a NanoDrop ${ }^{\mathrm{TM}}$ spectrophotometer (NanoDrop Technologies; Thermo Fisher Scientific, Inc.), which was then diluted to $500 \mathrm{ng} / \mu \mathrm{l}$. Total RNA was reverse transcribed into cDNA using a SuperScript ${ }^{\mathrm{TM}}$ II First-Strand cDNA synthesis kit (Invitrogen; Thermo Fisher Scientific, Inc.) according to the manufacturer's protocol. qPCR was subsequently performed using a QuantiFast SYBR Green PCR kit (cat. no. 204057; Qiagen, Inc.) according to the manufacturer's protocol. The following thermocycling conditions were used for the qPCR: Initial denaturation at $94^{\circ} \mathrm{C}$ for $2 \mathrm{~min} ; 35$ cycles of $94^{\circ} \mathrm{C}$ for $30 \mathrm{sec}, 63^{\circ} \mathrm{C}$ for $30 \mathrm{sec}, 72^{\circ} \mathrm{C}$ for $1 \mathrm{~min}$; and a final extension at $72^{\circ} \mathrm{C}$ for $7 \mathrm{~min}$, prior to being maintained at $4^{\circ} \mathrm{C}$. The following primer sequences used are listed in Table I. Expression levels were quantified using the $2^{-\Delta \Delta \mathrm{Cq}}$ method (21) and normalized to the loading controls, GAPDH or U6, for mRNA or miR-802 expression, respectively. 
Statistical analysis. Statistical analysis was performed using GraphPad Prism v6.01 software (GraphPad Software, Inc.) and data are presented as the mean $\pm \mathrm{SD}$ of $\geq 3$ independent experiments. Statistical differences between two groups were determined using a paired Student's t-test, whereas statistical differences among multiple groups were analyzed using a one-way ANOVA, followed by a Bonferroni's correction post hoc test. $\mathrm{P}<0.05$ was considered to indicate a statistically significant difference.

\section{Results}

miR-802 expression levels in cervical cancer tissues and cell lines. The expression levels of miR-802 were significantly decreased in the cervical cancer tissues compared with the adjacent tissue $(\mathrm{P}<0.001$; Fig. 1A). Similarly, the expression levels of miR-802 were observed to be significantly decreased in the cervical cancer cell lines (HeLa, C-33 A, SiHa and ME-180) compared with the EEC cell line ( $\mathrm{P}<0.001$; Fig. 1B).

Effect of the overexpression of miR-802 on cervical cancer cells. The expression levels of miR-802 were significantly increased following the transfection of the miR-802 mimic into SiHa cells compared with the control and NC-mimic groups $(\mathrm{P}<0.001 ;$ Fig. $2 \mathrm{~A})$. However, following the overexpression of miR-802 in the cells, no significant difference was observed in the cell viability compared with the control group ( $\mathrm{P}>0.05$; Fig. 2B). Moreover, a Transwell assay was performed to determine the migratory ability of SiHa cells; the results revealed that the overexpression of miR-802 significantly decreased the migratory rate compared with the control and NC-mimic groups $(\mathrm{P}<0.001$; Fig. $2 \mathrm{C}$ and $\mathrm{D})$. Similarly, the cell invasive ability was significantly inhibited in the miR-802 mimic-transfected cells compared with the control and NC-mimic groups $(\mathrm{P}<0.001$; Fig. 2E and F).

BTF3 is a direct target gene of miR-802. The possible target genes of miR-802 were predicted using TargetScan software and it was discovered that miR-802 bound to the 3'-UTR of BTF3 (Fig. 3A). Subsequently, two pmirGLO dual-luciferase reporter vectors, namely, BTF3-3'-UTR and BTF3-3'-UTR-mut, were constructed. BTF3-3'-UTR or BTF3-3'-UTR-mut were co-transfected with the miR-802 mimic into the cells. The miR-802+BTF-3'-UTR group displayed significantly decreased relative luciferase activity compared with the other two groups: therefore, the results indicated that BTF3 was a direct target gene of miR-802 ( $\mathrm{P}<0.001$; Fig. 3B). The expression levels of BTF3 in the pWZL-BTF3 group were significantly increased compared with the pWZL-NC and control groups, suggesting that the BTF3 overexpression transfections were successful $(\mathrm{P}<0.001$; Fig. 3D). Furthermore, the expression levels of miR-802 were significantly increased in the pWZL-BTF3 + miR-802 mimic group compared with the pWZL-BTF3 + NC-mimic group $(\mathrm{P}<0.001$; Fig. $3 \mathrm{C})$. The highest expression levels of BTF3 were observed in the pWZL-BTF3 + NC-mimic group, whilst the expression levels of BTF3 in the pWZL-BTF3 + miR-802 mimic group were significantly decreased compared with the pWZL-BTF3 + NC-mimic group, but significantly increased compared with the miR-802 mimic group $(\mathrm{P}<0.001$; Fig. 3E-G).
miR-802 suppresses cell migration, invasion and epithelial-mesenchymal transition (EMT) in cervical cancer cells through regulating BTF3 expression. The pWZL-BTF3 + NC-mimic group significantly increased cell viability compared with the pWZL-NC + NC-mimic group; however, co-transfection with the miR-802 mimic (pWZL-BTF3 + miR-802 mimic group) significantly reversed the effect of BTF3 on cell viability $(\mathrm{P}<0.001$; Fig. 4A). Moreover, the overexpression of BTF3 significantly increased the cell migratory rate compared with the pWZL-NC + NC-mimic group, whereas this effect was significantly inhibited following co-transfection with the miR-802 mimic $(\mathrm{P}<0.001$; Fig. 4B and C). Similarly, co-transfection with the miR-802 mimic significantly suppressed the function of BTF3 alone in promoting the cell invasive ability $(\mathrm{P}<0.001$; Fig. 4D and $\mathrm{E})$. In addition, the regulatory effect of miR-802 on the EMT process of cervical cancer cells was investigated. The results demonstrated that the pWZL-BTF3 + NC-mimic group displayed significantly increased mRNA and protein expression levels of MMP2, MMP9 and N-cadherin compared with the pWZL-NC + NC-mimic group, whist inhibiting the expression levels of E-cadherin expression; however, the co-transfection of cells with pWZL-BTF and miR-802 mimic significantly reversed the effects of BTF3 on these genes $(\mathrm{P}<0.001$; Fig. 4F-H).

\section{Discussion}

Due to cervical cytology screening being widely implemented worldwide, the incidence and mortality rates of cervical cancer are decreasing (22). In addition, the development and clinical application of prophylactic tumor vaccines against cervical cancer are hypothesized to be able to effectively control cervical cancer in the next 20 to 30 years (23). However, at present, the therapeutic strategies used to treat patients with cervical cancer are ineffective, especially for patients presenting with advanced metastasis (24). Therefore, it is urgent and of great significance to determine the molecular mechanisms behind the occurrence, development and metastasis of cervical cancer.

miR-802, which is a newly discovered endogenous single-stranded non-coding small RNA molecule, has been found to be able to regulate the occurrence and development of gastric cancer, breast cancer and tongue squamous cell carcinoma $(14,25,26)$. In fact, a previous study observed that miR-802 expression was increased in osteosarcoma cells compared with the adjacent normal tissues (27). Currently, the effects of miR-802 and cervical cancer have not been reported in detail; thus, the current study aimed to determine the mechanisms of action of miR-802 in cervical cancer tumors. Consistent with the expression levels in gastric cancer, breast cancer and tongue squamous cell carcinoma, it was revealed that miR-802 expression levels were decreased in cervical cancer tissues and cell lines compared with the control group.

Moreover, the effects of miR-802 on the biological activity of cervical cancer cells were investigated; it was discovered that the overexpression of miR-802 had no significant effect on the cell viability of cervical cancer cells, but it inhibited their invasive and migratory abilities, indicating that miR-802 may exert certain inhibitory effects over the regulation of 

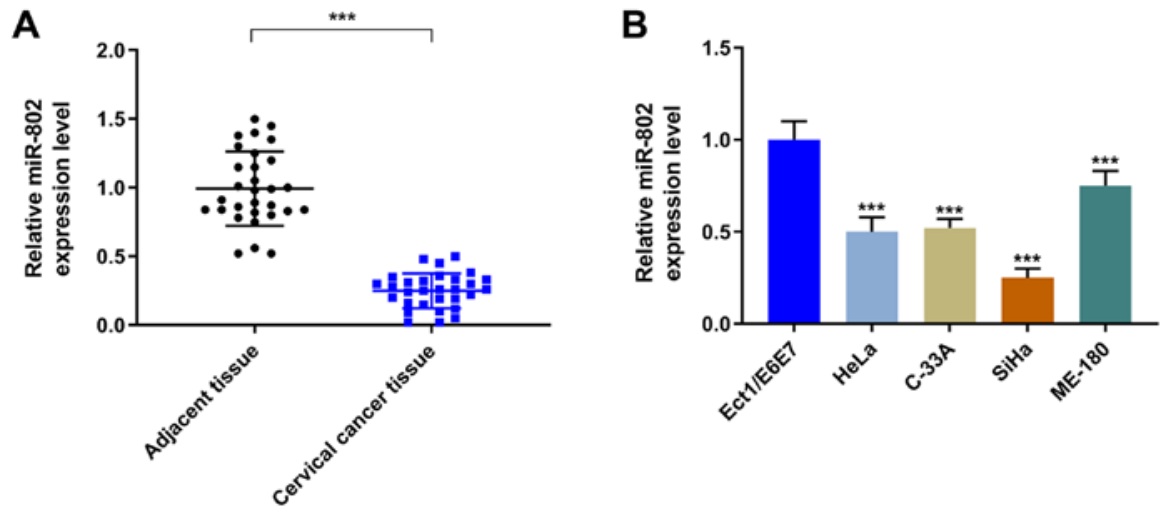

Figure 1. miR-802 expression in cervical cancer tissues and cell lines. (A) Expression levels of miR-802 in cervical cancer tissues and adjacent tissues was determined using RT-qPCR. N=3; ${ }^{* * *} \mathrm{P}<0.001$ vs. adjacent tissue. (B) RT-qPCR was performed to determine the expression levels of miR-802 in human EECs and cervical cancer cell lines (HeLa, C-33 A, SiHa and ME-180). N=3; ${ }^{* * *} \mathrm{P}<0.001$ vs. Ect1/E6E7. miR, microRNA; RT-qPCR, reverse transcription-quantitative PCR.

\section{A}
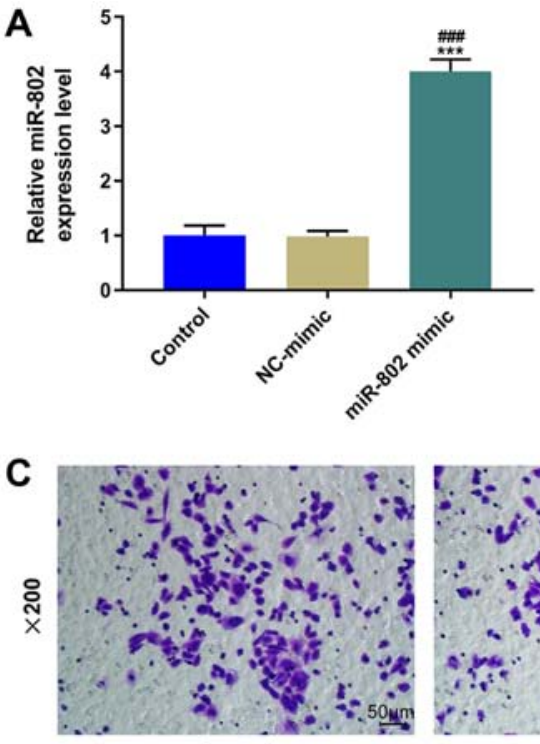

Control

D

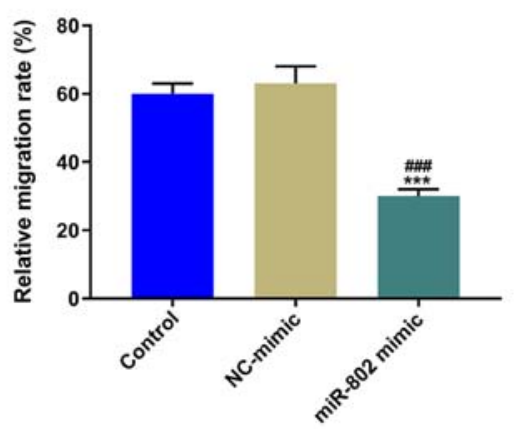

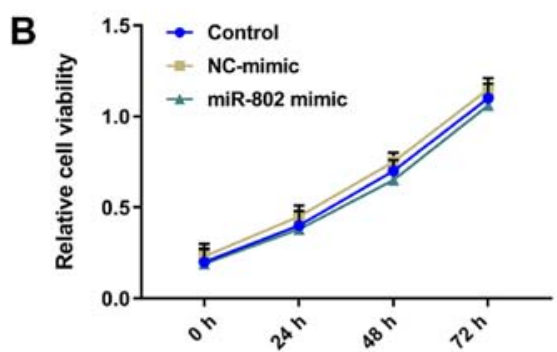

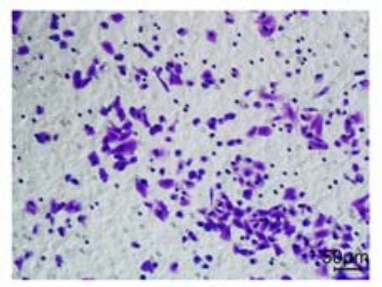

NC-mimic

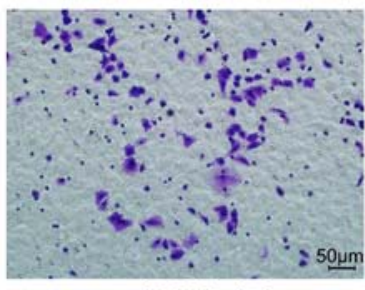

miR-802 mimic

E

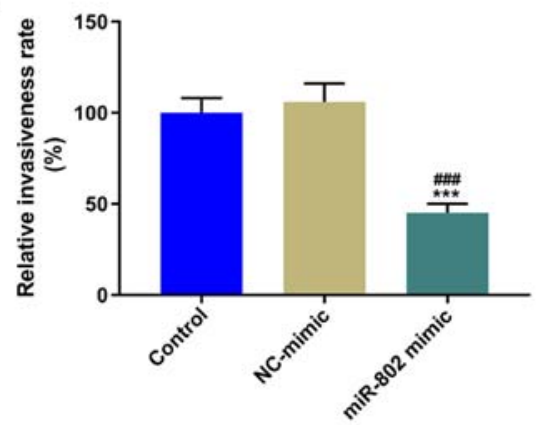

F
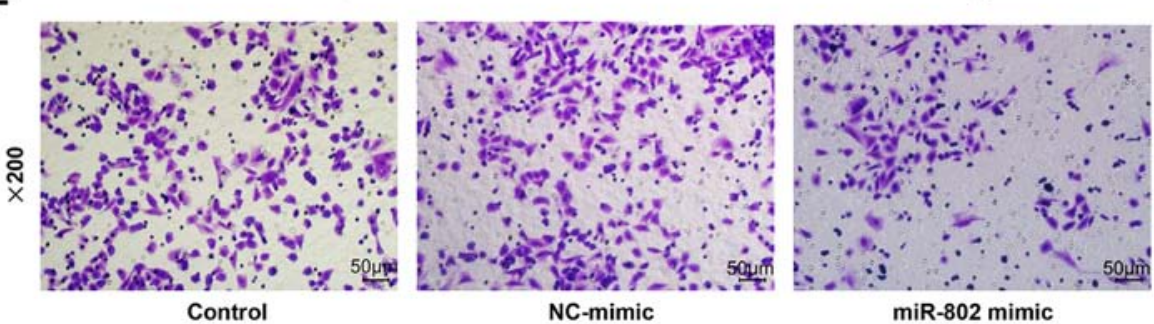

Figure 2. Effects of miR-802 overexpression on cervical cancer cells. (A) Reverse transcription-quantitative PCR was performed to determine the transfection efficiency of miR-802 in SiHa cells. U6 served as the internal reference gene. $\mathrm{N}=3$; ${ }^{* * *} \mathrm{P}<0.001$ vs. control; ${ }^{\# \# *} \mathrm{P}<0.001$ vs. NC-mimic. (B) Cell Counting Kit-8 assay was performed to analyze the effects of the miR-802 mimic on the viability of SiHa cells. (C and D) A Transwell assay was performed to determine the migratory ability of SiHa cells. Scale bar, $50 \mu \mathrm{m}$; magnification, $\mathrm{x} 200 ; \mathrm{n}=3 ;{ }^{* * * *} \mathrm{P}<0.001$ vs. control; ${ }^{\# \#} \mathrm{P}<0.001$ vs. NC-mimic. (E and $\mathrm{F}$ ) Invasive ability of SiHa cells was analyzed using a Transwell assay. Scale bar, $50 \mu \mathrm{m}$; magnification, $\mathrm{x} 200 ; \mathrm{n}=3 ;{ }^{* * * *} \mathrm{P}<0.001 \mathrm{vs}$. control; ${ }^{\# \# "} \mathrm{P}<0.001 \mathrm{vs}$. NC-mimic. miR, microRNA; NC, negative control. 
A

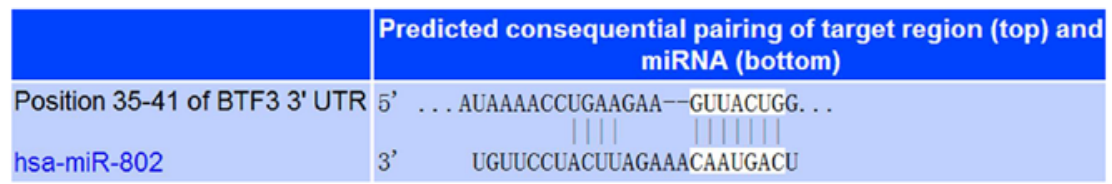

B

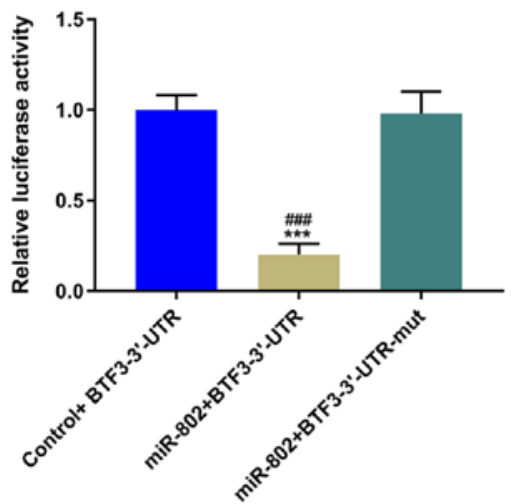

D

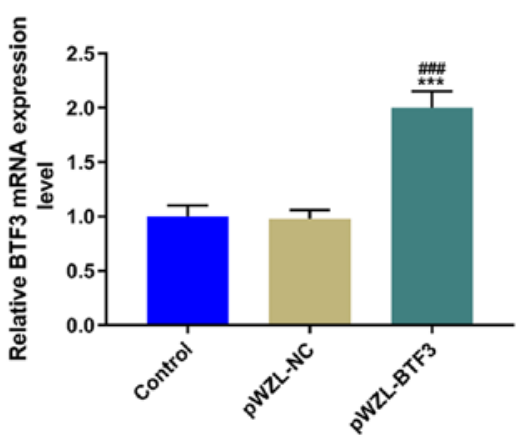

$\mathbf{F}$

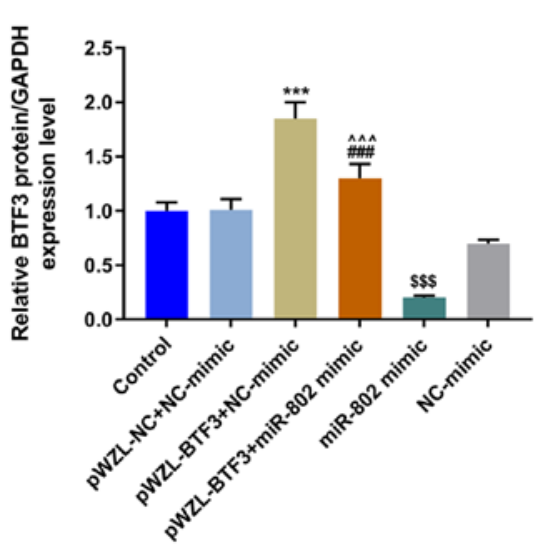

C

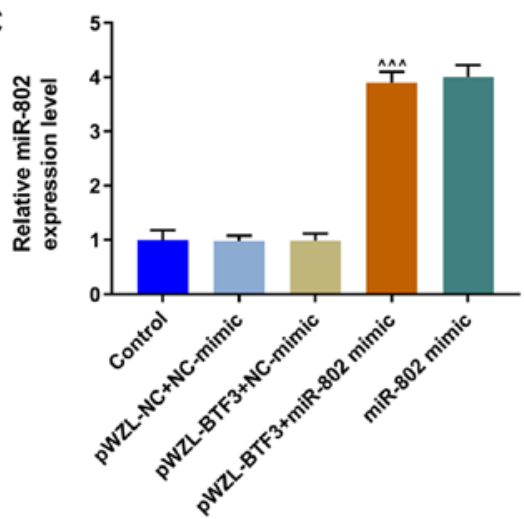

$\mathbf{E}$

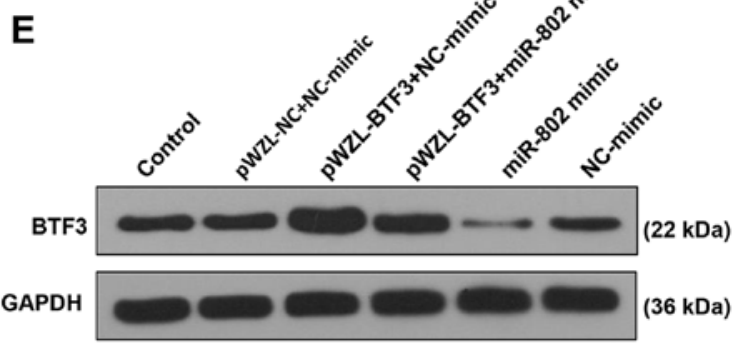

G

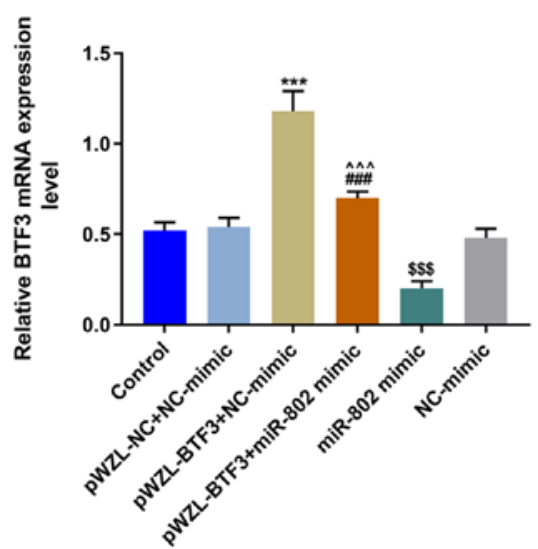

Figure 3. miR-802 directly targets BTF3 and its expression in cervical cancer cells. (A) TargetScan 7.2 software was used to predict the possible target genes of miR-802. (B) Dual-luciferase reporter assay was performed to determine the relative luciferase activity of BTF3. $N=3$; ${ }^{* * *} \mathrm{P}<0.001 \mathrm{vs}$. control + BTF3-3'-UTR;

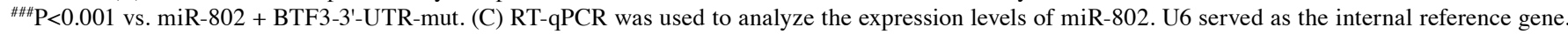
$\mathrm{N}=3 ;{ }^{\wedge \wedge} \mathrm{P}<0.001$ vs. pWZL-BTF3 + NC-mimic. (D) RT-qPCR was performed to detect the expression levels of BTF3. GAPDH served as the internal reference gene. $\mathrm{N}=3 ;{ }^{* * *} \mathrm{P}<0.001$ vs. control; ${ }^{\# \# /} \mathrm{P}<0.001$ vs. pWZL-NC. (E and $\mathrm{F}$ ) Western blotting was performed to determine the expression levels of $\mathrm{BTF}$. N=3; ${ }^{* * * * *} \mathrm{P}<0.001$ vs. pWZL-NC $+\mathrm{NC}-\mathrm{mimic} ;{ }^{\# \# \prime \prime} \mathrm{P}<0.001$ vs. pWZL-BTF3 + NC-mimic; ${ }^{\wedge \wedge} \mathrm{P}<0.001$ vs. miR-802 mimic; sssP $<0.001$ vs. NC-mimic. (G) RT-qPCR was performed to analyze the expression levels of BTF3. GAPDH served as the internal reference gene. $\mathrm{N}=3 ;{ }^{* * *} \mathrm{P}<0.001 \mathrm{vs}$. pWZL-NC $+\mathrm{NC}-\mathrm{mimic}$; ${ }^{* \# \#} \mathrm{P}<0.001 \mathrm{vs}$. pWZL-BTF3 + NC-mimic; ${ }^{\wedge \wedge} \mathrm{P}<0.001$ vs. miR-802 mimic; ${ }^{\$ \$} \mathrm{P}<0.001$ vs. NC-mimic. miR, microRNA; NC, negative control; BTF3, basic transcription factor 3; RT-qPCR, reverse transcription-quantitative PCR.

cervical cancer cells. Furthermore, miR-802 was reported to promote osteosarcoma cell proliferation by targeting p27 (27). A previous study also found that miR-802 promoted the proliferation of lung cancer cells through negatively regulating the tumor suppressor menin (28), and inhibited the invasion and migration of gastric cancer cells, whilst promoting cell apoptosis by targeting RAB23 (14).
TargetScan 7.2 software was used to predict that the signal transductor and transcriptional activator BTF3 was a potential target gene of miR-802; this was further validated in the present study using a dual-luciferase reporter assay, whereby miR-802 inhibited the activity of the BTF3 3'-UTR reporter gene. Several studies have previously reported that BTF3 regulated tumor cell migration and invasion (29-31); for 
A

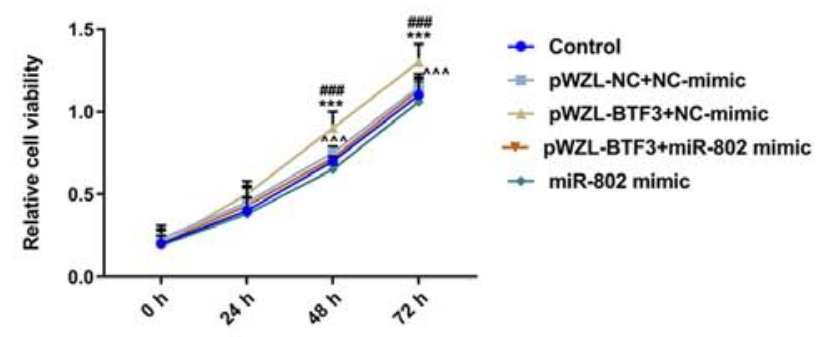

B

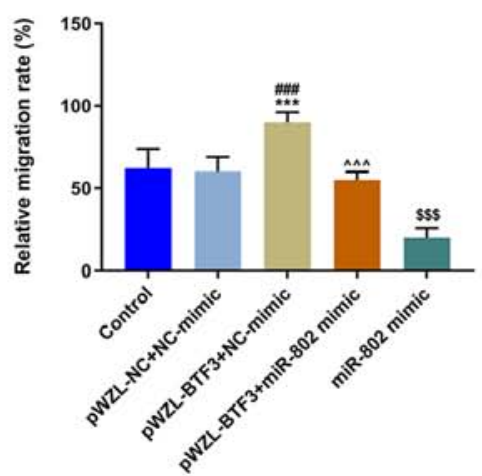

C
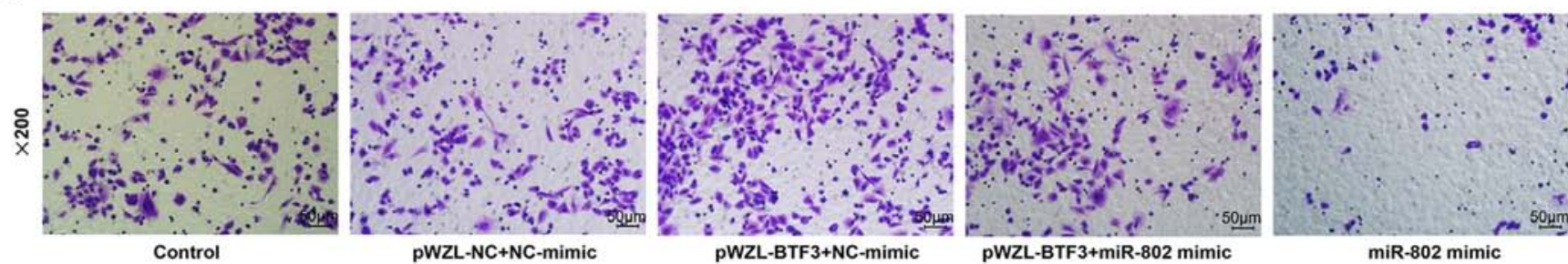

D
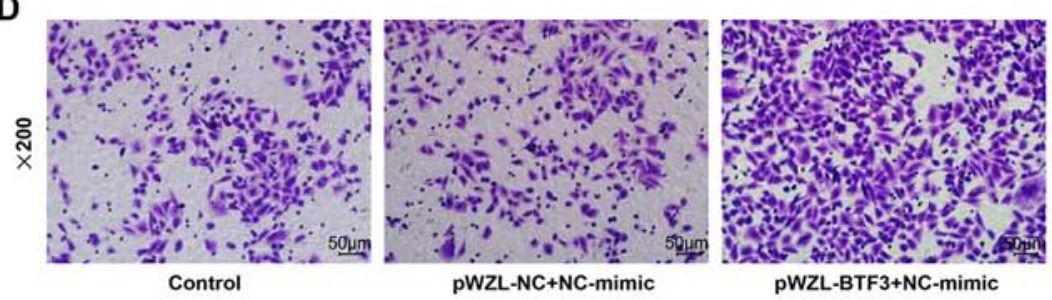

PWZL-BTF3+NC-mimic

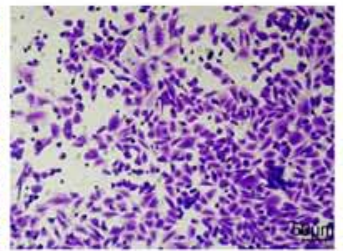

PWZL-BTF3+miR-802 mimic

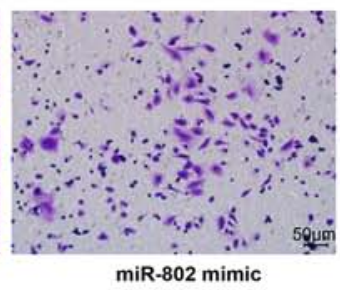

E

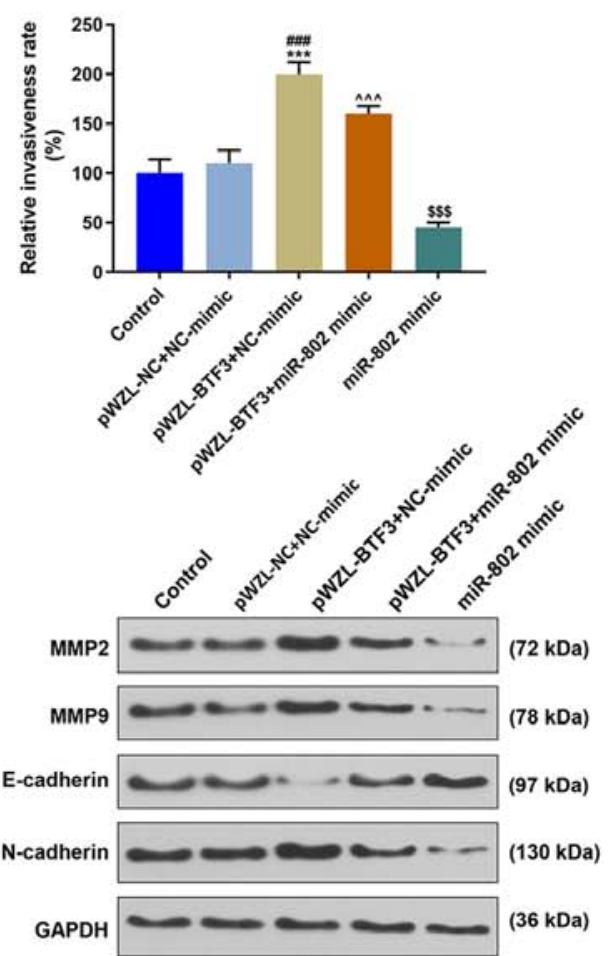

$\mathbf{F}$

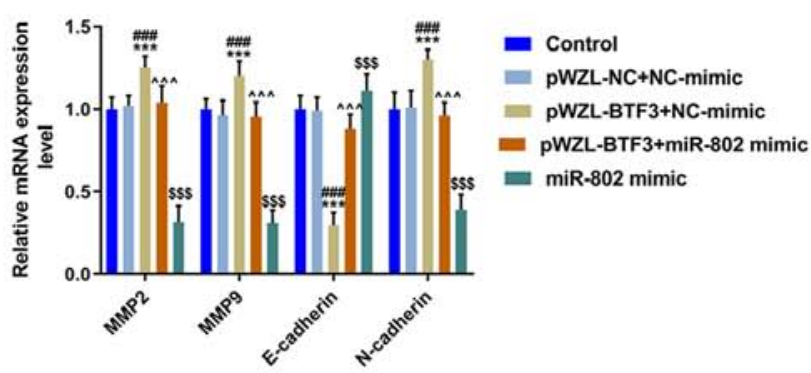

H

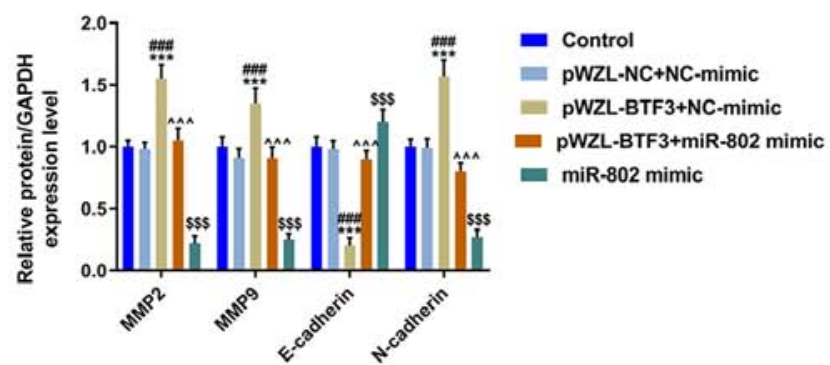

Figure 4. miR-802 suppresses cell migration, invasion and epithelial-mesenchymal transition in cervical cancer cells through regulating BTF3 expression. (A) Cell Counting Kit- 8 assay was performed to detect the viability of SiHa cells. $n=3 ;{ }^{* * *} \mathrm{P}<0.001$ vs. control; ${ }^{\# \# \#} \mathrm{P}<0.001$ vs. pWZL-NC $+\mathrm{NC}-\mathrm{mimic} ;{ }^{\wedge \wedge} \mathrm{P}<0.001$ vs. pWZL-BTF3 + NC-mimic. (B and C) Transwell assay was performed to determine the migratory ability of SiHa cells. Scale bar, $50 \mu \mathrm{m}$; magnification, $\mathrm{x} 200 ; \mathrm{n}=3 ;{ }^{* * *} \mathrm{P}<0.001$ vs. control; ${ }^{\# \# \#} \mathrm{P}<0.001$ vs. pWZL-NC + NC-mimic; ${ }^{\wedge \wedge} \mathrm{P}<0.001$ vs. pWZL-BTF3 + NC-mimic; ${ }^{\$ \$ \$} \mathrm{P}<0.001$ vs. pWZL-BTF3 + miR-802 mimic. (D and E) Invasive ability of SiHa cells was analyzed using a Transwell assay. Scale bar, $50 \mu \mathrm{m}$; magnification, $\mathrm{x} 200 ; \mathrm{n}=3 ;{ }^{* * *} \mathrm{P}<0.001 \mathrm{vs}$. control;

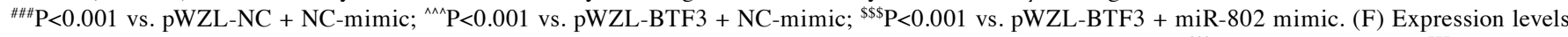
of MMP9, MMP2, E-cadherin and N-cadherin were detected using reverse transcription-quantitative $\mathrm{PCR}$. N=3; ${ }^{* * *} \mathrm{P}<0.001$ vs. control; ${ }^{\# \# \#} \mathrm{P}<0.001 \mathrm{vs}$. pWZL-NC + NC-mimic; ${ }^{\wedge \wedge} \mathrm{P}<0.001$, vs. pWZL-BTF3 + NC-mimic; ${ }^{\$ \$ S} \mathrm{P}<0.001$ vs. pWZL-BTF3 + miR-802 mimic. (G and H) Western blotting was used to analyze the expression levels of MMP9, MMP2, E-cadherin and N-cadherin. $\mathrm{N}=3 ;{ }^{* * * *} \mathrm{P}<0.001$ vs. control; ${ }^{\# \# "} \mathrm{P}<0.001$ vs. pWZL-NC $+\mathrm{NC}-\mathrm{mimic} ;{ }^{\wedge \wedge} \mathrm{P}<0.001$ vs. pWZL-BTF3 + NC-mimic; ${ }^{\$ \$}$ P $<0.001$ vs. pWZL-BTF3 + miR-802 mimic. GAPDH served as the internal reference control. miR, microRNA; NC, negative control; BTF3, basic transcription factor 3; MMP, matrix metallopeptidase. 
example, Zhang et al (32) reported that the genetic knockdown of BTF3 impaired the regulation of proliferation, the cell cycle and apoptosis in hypopharyngeal squamous cell carcinoma via the serine-protein kinase ATM signaling pathway (32). BTF3 also promoted prostate cancer progression through modeling stem-like traits (33). These studies indicated that BTF3 may have a role in promoting cancer. Thus, the roles of miR-802 and BTF3 in cervical cancer cells was further investigated and it was revealed that overexpressing miR-802 significantly reversed the function of BTF3 in promoting the cell viability, migration and invasion.

In addition, EMT refers to the process via which epithelial cells acquire invasive mesenchymal phenotypes and it is considered as an important mechanism in the initial stages of tumor cell metastasis (34). EMT is closely related to the occurrence and development of cervical cancer tumors; therefore, it may be an important target for the treatment of cervical cancer (35). Studies have increasingly demonstrated that miRNAs are important regulatory factors related to EMT; for instance, in cervical cancer, miR-145 and miR-1 inhibited the invasion and migration of cancer cells through regulating EMT (36,37). Zhang et al (38), also reported that knocking down BTF3 expression increased E-cadherin expression, but decreased $\mathrm{N}$-cadherin and ZEB2 expression, which had an overall effect of downregulating EMT processes in gastric cancer (38). In the current study, the overexpression of BTF3 increased the expression levels of N-cadherin and inhibited those of E-cadherin. Notably, the protein expression levels of E-cadherin were increased in the cells which overexpressed miR-802, suggesting that miR-802 may suppress EMT in cervical cancer through targeting BTF3. In addition, MMP2 and MMP9 are considered as proteins related to tumor metastasis, and it was previously reported that decreasing their expression levels inhibited the migration and progression of cancer cells (39). In the present study, the overexpression of miR-802 markedly suppressed the expression levels of MMP2 and MMP9 in cells by regulating BTF3 expression.

However, this study also has several limitations; for example, the results obtained would be more convincing if multiple cervical cancer cell lines were used in the study to investigate the role of miR-802 in cervical cancer. In addition, the present study did not include in vivo experiments, which could verify miR-802 targeting of BTF3.

In conclusion, the findings of the current study suggested that the overexpression of miR-802 may suppress cervical cancer progression by decreasing BTF3 expression levels, indicating that miR-802 may be a potential therapeutic target for the treatment and prognosis of patients with cervical cancer.

\section{Acknowledgements}

Not applicable.

\section{Funding}

The present study was supported by the Jingmen General Science and Technology Project of Hubei Province (grant no. 2019YFYB022).

\section{Availability of data and materials}

The datasets used/or analyzed during the study are available from the corresponding author on reasonable request.

\section{Authors' contributions}

XW and LL conceived and designed the study, drafted the manuscript and critically revised it for important intellectual content. HZ acquired the data, performed the data analysis and interpreted the results. All authors read and approved the final manuscript and agreed to be accountable for all aspects of the work in ensuring that questions related to the accuracy or integrity of the work are appropriately investigated and resolved.

\section{Ethics approval and consent to participate}

All research subjects provided written informed consent and the study was approved by the Ethics Committees of Jingmen First People's Hospital. All procedures performed in the studies involving human participants were in accordance with the ethical standards of the institutional and/or national research committee, and with the 1964 Declaration of Helsinki and its later amendments or comparable ethical standards.

\section{Patient consent for publication}

Not applicable.

\section{Competing interests}

The authors declare that they have no competing interests.

\section{References}

1. Fang J, Zhang $\mathrm{H}$ and Jin S: Epigenetics and cervical cancer: From pathogenesis to therapy. Tumour Biol 35: 5083-5093, 2014.

2. Dehn D, Torkko KC and Shroyer KR: Human papillomavirus testing and molecular markers of cervical dysplasia and carcinoma. Cancer 111: 1-14, 2007.

3. Sun XL, Wang HB, Wang ZQ, Cao TT, Yang X, Han JS, Wu YF, Reilly KH and Wang JL: Effect of transcutaneous electrical stimulation treatment on lower urinary tract symptoms after class III radical hysterectomy in cervical cancer patients: Study protocol for a multicentre, randomized controlled trial. BMC Cancer 17: 416, 2017.

4. Kazumoto T, Kato S, Yokota H, Hasumi Y, Kino N, Horie K, Yoshida D, Mizukami T and Saito Y: Is a low dose of concomitant chemotherapy with extended-field radiotherapy acceptable as an efficient treatment for cervical cancer patients with metastases to the para-aortic lymph nodes? Int J Gynecol Cancer 21: 1465-1471, 2011.

5. Xue M, Zhuo Y and Shan B: MicroRNAs, long noncoding RNAs, and their functions in human disease. Methods Mol Biol 1617: 1-25, 2017.

6. Mannucci C, Casciaro M, Minciullo PL, Calapai G, Navarra M and Gangemi S: Involvement of microRNAs in skin disorders: A literature review. Allergy Asthma Proc 38: 9-15, 2017.

7. Song $\mathrm{Z}$ and Li G: Role of specific microRNAs in regulation of vascular smooth muscle cell differentiation and the response to injury. J Cardiovasc Transl Res 3: 246-250, 2010.

8. Singh RP, Massachi I, Manickavel S, Singh S, Rao NP, Hasan S, Mc Curdy DK, Sharma S, Wong D, Hahn BH, et al: The role of miRNA in inflammation and autoimmunity. Autoimmun Rev 12: 1160-1165, 2013.

9. Xu YF, Mao YP, Li YQ, Ren XY, He QM, Tang XR, Sun Y, Liu N and Ma J: MicroRNA-93 promotes cell growth and invasion in nasopharyngeal carcinoma by targeting disabled homolog-2. Cancer Lett 363: 146-155, 2015. 
10. Paul P, Chakraborty A, Sarkar D, Langthasa M, Rahman M, Bari M, Singha RS, Malakar AK and Chakraborty S: Interplay between miRNAs and human diseases. J Cell Physiol 233. 2007-2018, 2018

11. Chen YL, Xu QP, Guo F and Guan WH: MicroRNA-302d downregulates TGFBR2 expression and promotes hepatocellular carcinoma growth and invasion. Exp Ther Med 13: 681-687, 2017

12. Wang F, Liu J, Zou Y, Jiao Y, Huang Y, Fan L, Li X, Yu H, He C, Wei W, etal: MicroRNA-143-3p, up-regulated in H.pylori-positive gastric cancer, suppresses tumor growth, migration and invasion by directly targeting AKT2. Oncotarget 8: 28711-28724, 2017.

13. Li N and Qin ZB: Inflammation-induced miR-802 promotes cell proliferation in cholesteatoma. Biotechnol Lett 36: 1753-1759, 2014.

14. Zhang XY, Mu JH, Liu LY and Zhang HZ: Upregulation of miR-802 suppresses gastric cancer oncogenicity via targeting RAB23 expression. Eur Rev Med Pharmacol Sci 21: 4071-4078, 2017.

15. Zhang Q, Lv R, Guo W and Li X: microRNA-802 inhibits cell proliferation and induces apoptosis in human cervica cancer by targeting serine/arginine-rich splicing factor 9 . J Cell Biochem 120: 10370-10379, 2019.

16. Delfino KR and Rodriguez-Zas SL: Transcription factor-microRNA-target gene networks associated with ovarian cancer survival and recurrence. PLoS One 8: e58608, 2013.

17. Zhou W, Wang Y, Wu R, He Y, Su Q and Shi G: MicroRNA-488 and -920 regulate the production of proinflammatory cytokines in acute gouty arthritis. Arthritis Res Ther 19: 203, 2017.

18. Hu J, Ni S, Cao Y, Zhang T, Wu T, Yin X, Lang Y and Lu H: The angiogenic effect of microRNA-21 targeting TIMP3 through the regulation of MMP2 and MMP9. PLoS One 11: e0149537, 2016.

19. Liu GW, Qin ZM and Shen QH: An ensemble method integrated with miRNA expression data for predicting miRNA targets in stomach adenocarcinoma. Cancer Biomark 20: 617-625, 2017.

20. Li Y, Cai B, Shen L, Dong Y, Lu Q, Sun S, Liu S, Ma S, Ma PX and Chen J: MiRNA-29b suppresses tumor growth through simultaneously inhibiting angiogenesis and tumorigenesis by targeting Akt3. Cancer Lett 397: 111-119, 2017.

21. Livak KJ and Schmittgen TD: Analysis of relative gene expression data using real-time quantitative PCR and the 2(-Delta Delta C(T)) Method. Methods 25: 402-408, 2001.

22. Cakmak B and Köseoğlu DR: Comparison of cervical cytological screening results between postmenopausal and elderly women. Turk Patoloji Derg 30: 38-42, 2014.

23. Cordeiro MN, De Lima RCP, Paolini F, Melo ARDS Campos APF, Venuti A and De Freitas AC: Current research into novel therapeutic vaccines against cervical cancer. Expert Rev Anticancer Ther 18: 365-376, 2018.

24. Favero G, Pierobon J, Genta ML, Araujo MP, Miglino G, Del Carmen Pilar Diz M, de Andrade Carvalho H, Fukushima JT, Baracat EC and Carvalho JP: Laparoscopic extrafascial hysterectomy (completion surgery) after primary chemoradiation in patients with locally advanced cervical cancer: technical aspects and operative outcomes. Int J Gynecol Cancer 24: 608-614, 2014.

25. Yuan F and Wang W: MicroRNA-802 suppresses breast cancer proliferation through downregulation of FoxM1. Mol Med Rep 12: 4647-4651, 2015.
26. Wu X, Gong Z, Sun L, Ma L and Wang Q: MicroRNA-802 plays a tumour suppressive role in tongue squamous cell carcinoma through directly targeting MAP2K4. Cell Prolif 50: 50, 2017.

27. Cao ZQ, Shen Z and Huang WY: MicroRNA-802 promotes osteosarcoma cell proliferation by targeting p27. Asian Pac J Cancer Prev 14: 7081-7084, 2013.

28. Wang LQ, Chen G, Liu XY, Liu FY, Jiang SY and Wang Z: microRNA-802 promotes lung carcinoma proliferation by targeting the tumor suppressor menin. Mol Med Rep 10: 1537-1542, 2014.

29. Symes AJ, Eilertsen M, Millar M, Nariculam J, Freeman A, Notara M, Feneley MR, Patel HR, Masters JR and Ahmed A: Quantitative analysis of BTF3, HINT1, NDRG1 and ODC1 protein over-expression in human prostate cancer tissue. PLoS One 8: e84295, 2013

30. Wang CJ, Frånbergh-Karlson H, Wang DW, Arbman G, Zhang H and Sun XF: Clinicopathological significance of BTF3 expression in colorectal cancer. Tumour Biol 34: 2141-2146, 2013.

31. Ding J, Wang X, Zhang Y, Sang X, Yi J, Liu C, Liu Z, Wang M, Zhang N, Xue Y, et al: Inhibition of BTF3 sensitizes luminal breast cancer cells to PI3K $\alpha$ inhibition through the transcriptional regulation of ER $\alpha$. Cancer Lett 440-441: 54-63, 2019.

32. Zhang Y, Gross N, Li Z, Yin G, Zhong Q, Liu C and Huang Z: Upregulation of BTF3 affects the proliferation, apoptosis, and cell cycle regulation in hypopharyngeal squamous cell carcinoma. Biomed Pharmacother 118: 109211, 2019.

33. Hu J, Sun F, Chen W, Zhang J, Zhang T, Qi M, Feng T, Liu H, Li X, Xing Y, et al: BTF3 sustains cancer stem-like phenotype of prostate cancer via stabilization of BMI1. J Exp Clin Cancer Res 38: 227, 2019.

34. Hanahan D and Weinberg RA: Hallmarks of cancer: The next generation. Cell 144: 646-674, 2011.

35. Qureshi R, Arora H and Rizvi MA: EMT in cervical cancer: Its role in tumour progression and response to therapy. Cancer Lett 356 (2 Pt B): 321-331, 2015.

36. Sathyanarayanan A, Chandrasekaran KS and Karunagaran D: microRNA-145 modulates epithelial-mesenchymal transition and suppresses proliferation, migration and invasion by targeting SIP1 in human cervical cancer cells. Cell Oncol (Dordr) 40: 119-131, 2017.

37. Cheng Y, Yang M and Peng J: Correlation the between the regulation of miRNA-1 in c-Met-induced EMT and cervical cancer progression. Oncol Lett 17: 3341-3349, 2019.

38. Zhang DZ, Chen BH, Zhang LF, Cheng MK, Fang XJ and Wu XJ: Basic transcription factor 3 is required for proliferation and epithelial-mesenchymal transition via regulation of FOXM1 and JAK2/STAT3 signaling in gastric cancer. Oncol Res 25: 1453-1462, 2017

39. Liu H, Zeng Z, Wang S, Li T, Mastriani E, Li QH, Bao HX, Zhou YJ, Wang X, Liu Y, et al: Main components of pomegranate, ellagic acid and luteolin, inhibit metastasis of ovarian cancer by down-regulating MMP2 and MMP9. Cancer Biol Ther 18: 990-999, 2017.

This work is licensed under a Creative Commons Attribution-NonCommercial-NoDerivatives 4.0 International (CC BY-NC-ND 4.0) License. 